\title{
Optimizing Energy Conservation Measures in a Grocery Store using Present and Future Weather Files
}

\author{
Arfa N. Aijazi ${ }^{1}$, Rob Best ${ }^{2}$, Stefano Schiavon ${ }^{1}$ \\ ${ }^{1}$ Center for the Built Environment, University of California, Berkeley, USA \\ ${ }^{2}$ Arup, San Francisco, USA
}

\begin{abstract}
Grocery stores are one of the most energy intensive building types, which makes targets for zero net energy (ZNE) particularly challenging. This study builds on a prior computational optimization study to identify combinations of energy conservation measures (ECMs) for an existing grocery store in San Francisco. As the climate changes, also the retrofit recommendations based on simulation results from historical-based weather files may vary. In this paper, we looked at how the optimization results change when accounting for climate changes over the building's service life by using future weather files. We found that the expected changes in future weather are sufficient to alter retrofit recommendations. This type of analysis is thus important to ensure that buildings designed now can continue to meet performance objectives into the future.
\end{abstract}

\section{Introduction}

As illustrated by the Intergovernmental Panel on Climate Change's (IPCC) special report SR 15, the building industry is critical for mitigating climate change. All pathways that maintain a global temperature increase of $1.5^{\circ} \mathrm{C}$ require (a) building emissions to be reduced by 80 $90 \%$ by 2050 , (b) new construction to be fossil free and near-zero energy by 2020, and (c) energy refurbishment of the existing building stock by at least $5 \%$ per annum in developed countries (de Coninck, et al., 2018). To meet this challenge, the State of California requires that all new and $50 \%$ of existing commercial buildings be Zero Net Energy (ZNE) by 2030 (CARB, 2017; CEC, 2007).

ZNE can be challenging to achieve cost effectively in existing buildings because many design elements, like geometry, are already locked-in, thus limiting the scope of potential energy conservation measures (ECM). Achieving $\mathrm{ZNE}$ is also challenging for grocery stores because of the high energy use intensity (EUI) inherent to this building type. Prior studies demonstrate that it is possible to achieve ZNE for new grocery stores where manipulation of the building form is possible (HachemVermette, Cubi, and Bergerson, 2016; Arup, 2012; Acha et al., 2013). Further, the EUI must be offset by large areas of on-site renewable energy generation, such as on roofs, facades, and covered parking lots or off-site renewables (Pless and Torcellini, 2010). This can be especially challenging for buildings in dense urban areas.
The building at the heart of this project is the existing Whole Foods Market in the Noe Valley neighborhood of San Francisco, CA. The building exemplifies all the above challenges in that it is an existing grocery store in an urban area. The overall project goal to demonstrate the feasibility of ZNE despite these issues. The interdisciplinary project team consists of Prospect Silicon Valley, Whole Foods Market, Arup with funding from the California Energy Commission.

As part of the design phase, Arup developed a calibrated building energy model of the existing grocery store and applied computational optimization techniques to select from thousands of possible ECM combinations that met the project budget constraint of $\$ 2,000,000$ USD (Best and Levine, 2018). The single best design achieved an energy savings of $65 \%$ from the existing building but was unable to meet $\mathrm{ZNE}$ due to area limitations for on-site renewable energy generation.

One of the most important inputs for building energy models is the weather file, which impacts calculation of heating and cooling loads, and lighting energy requirements. Because of the high variability of measured weather data, conventional practice is to use synthetic weather files, such as Typical Meteorological Year (TMY), which are based on the historical weather record. However, observed global warming trends undermine the validity of this practice. Buildings designed and constructed today will experience climate-related changes over the course of their service life. This suggests that proper assessment of building performance should include simulations using projections of future weather. Especially for buildings evaluating retrofit strategies to meet climate goals, assessment using future weather files is important to understand impacts to ECM impact, feasibility, and cost-effectiveness as the climate changes.

The underlying question of this paper is how do anticipated changes in future weather impact our recommendations today on optimal ECMs? If we find significant changes, then the current standard practice of using only TMY weather files may be setting up buildings for suboptimal performance in the future.

\section{Methods}

To test these questions, we evaluated the calibrated model of the Whole Foods store with current and future weather. As part of our process, we applied an 
optimization routine to identify high performing combinations of ECMs

\section{Base Building Energy Model}

Arup developed the baseline building energy model in EnergyPlus v8.6. The model incorporates the existing building geometry and a detailed end-use build-up of all internal electrical loads, HVAC system distribution, and observed operating parameters from in-store monitoring. Arup tuned uncertain model parameters including schedules, refrigeration system operation, and additional unreported plug loads using 15-minute electricity and monthly gas utility data (Best and Levine, 2018). Arup calibrated the model in accordance to tolerances suggested by ASHRAE Guideline 14-2014: Measurement of Energy, Demand and Water Savings, resulting in a final calibrated model with a normalized mean bias error (NMBE) of less than $4 \%$ and a coefficient of variation of the root mean square error CV[RMSE] less than $11 \%$ for electricity and natural gas.

\section{ECM Selection}

The project team determined potential ECMs based on: 1) a design charrette with industry experts including a "blue sky" brainstorming session, 2) a site visit and kitchen equipment audit, 3) analyzed energy consumption data, and 4) a technology discovery competition. Together, these approaches generated a list of over 350 ECMs that the team reduced to 107 unique measures based on feasibility that were carried forward for modeling and cost analysis. Arup implemented these 107 ECMs within EnergyPlus by identifying mutually exclusive options and systems with parametric possibilities (i.e., insulation thickness) to create 58 unique pieces of equipment and systems, each with 1 to 19 parametric possibilities. These 58 ECM categories with their variants form the design space of possible retrofit options for this study. A full list of ECMs and descriptions can be found in (Best and Levine, 2018).

\section{Computational Optimization}

Identifying deep energy savings within a fixed capital budget, especially with interacting systems, requires assessing packages of measures rather than individual ECMs (Pless and Torcellini, 2010; O’Brien, Athienitis, and Kesik, 2011). However, with a large number of ECMs, enumeration of all combinations is computationally infeasible. We employed a genetic algorithm (GA) to efficiently evaluate different combinations of ECMs and identify the best opportunities for energy savings within the capital budget. This approach has been used previously for net-zero and energy efficient building design due to its ability to operate on problems with nonlinear relationships between variables and objectives (Wang, Zmeureanu, and Rivard, 2005; Tuhus-Dubrow and Krarti, 2010; Evins, 2013).

We defined the objective of the GA to minimize operational energy cost as well as three constraints. First, the capital cost of all ECMs could not exceed the $\$ 2,000,000$ USD construction budget. Second, we prevented mutually exclusive ECMs (e.g. HVAC system types) from inclusion in the same model. Third, we required any ECMs built on a DC (direct current) microgrid for the store to be implemented together to maximize the value of the microgrid.

We encoded the optimization in a Python script in which we selected ECMs for each major equipment or system from a discrete set of options using integer variables, and direct edits were made to the EnergyPlus file. Each combined test file was run for a four-month period (February, May, September, and November). We allowed the GA to run for 51 generations of 50 ECM packages per generation for a total of 2,448 successful packages of ECMs. We repeated this process for each weather file. Best and Levine describe the building energy model and optimization process in more detail elsewhere (Best and Levine, 2018).

\section{Future Weather}

We obtained future weather files from WeatherShift, an online tool developed by Arup and Argos Analytics to morph present TMY weather files to account for climate change (Dickinson and Brannon, 2016). Modeling future weather requires consideration of uncertainty in future GHG emissions. In the Fifth Assessment Report (AR5), the IPCC defines a set of four emissions scenarios, called Representative Concentration Pathways (RCP): RCP 2.5, RCP 4.5, RCP 6.0, and RCP 8.5. Emissions scenarios capture the range of possible human impact on future GHG emissions given factors such as population growth, economic development, technological innovation, and policy interventions. The RCP numbers refer to radiative forcing values, i.e. the difference between incoming insolation absorbed by the Earth and energy radiated back to space, in 2100 relative to pre-industrial levels in $\mathrm{W} / \mathrm{m}^{2}$. Lower levels of radiative forcing correspond to lower GHG emissions and concentrations. The four RCP pathways span the range of radiative forcing levels found in the literature.

This study compares results from two future emissions scenarios, RCP 4.5, which roughly corresponds to the objectives of the Paris Climate Agreement signed in 2014, and RCP 8.5, which is often dubbed "business as usual". Together, the two pathways represent realistic lower and upper bounds on future GHG emissions.

From GHG emissions estimates, climate scientists use numerical models to simulate interactions between atmospheric and oceanic processes at a global scale, called general circulation models (GCM). There can be lot of variation across climate models because they involve stochastic processes and are highly dependent on initial conditions. WeatherShift uses an ensemble of 14 GCM and ranks them based on projected changes in weather outputs to obtain a percentile distribution, called warming percentile. For this study, we used the median $\left(50^{\text {th }}\right.$ percentile) changes in weather outputs.

\section{Results and Discussion}

The results below compare outcomes from repeating the computational optimization procedure wherein the only change was the weather file for the building energy simulations. 


\section{Future Weather}

Before analyzing optimization results, we reviewed differences between the present TMY3 weather file and both future scenarios. Figure 1 compares the mean monthly dry bulb temperature (DBT). In the future the mean monthly DBT increases by between $1.5-3{ }^{\circ} \mathrm{C}$.

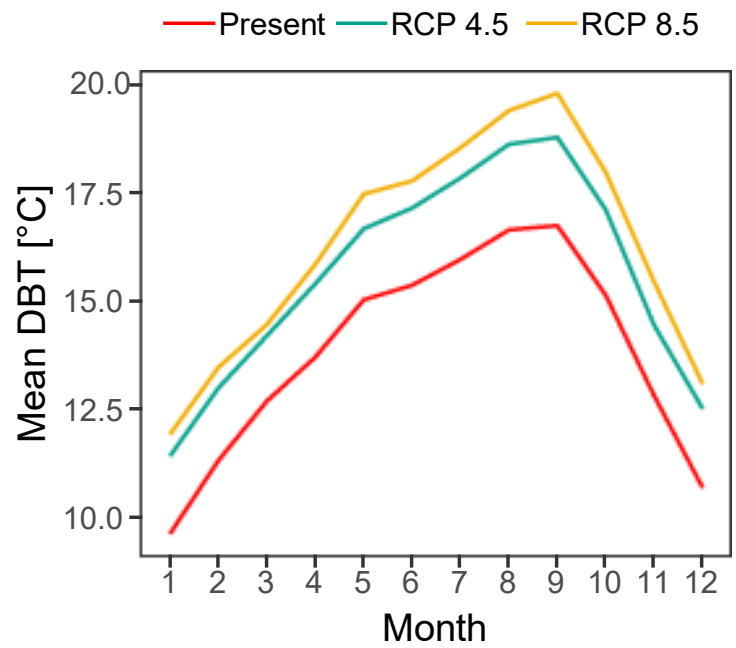

Figure 1: Mean monthly dry-bulb temper in present and future weather scenarios for San Francisco

To begin to understand the impact of this temperature change on building energy consumption, we calculated the heating and cooling degrees, HDD and CDD respectively, for the present and future, with a base temperature of $18.5{ }^{\circ} \mathrm{C}$. The results, plotted in Figure 2, show that San Francisco is currently a heating dominated climate with very few CDD. Under the RCP 4.5 emissions scenario, HDDs decrease by around $1 / 3$ but CDD increase 4-fold. Under RCP 8.5 HDD decrease by around $1 / 2$ but CDD increase nearly 10 -fold. The optimization results will show how significant these weather changes are for decisions on cost-effective energy conservation measures.

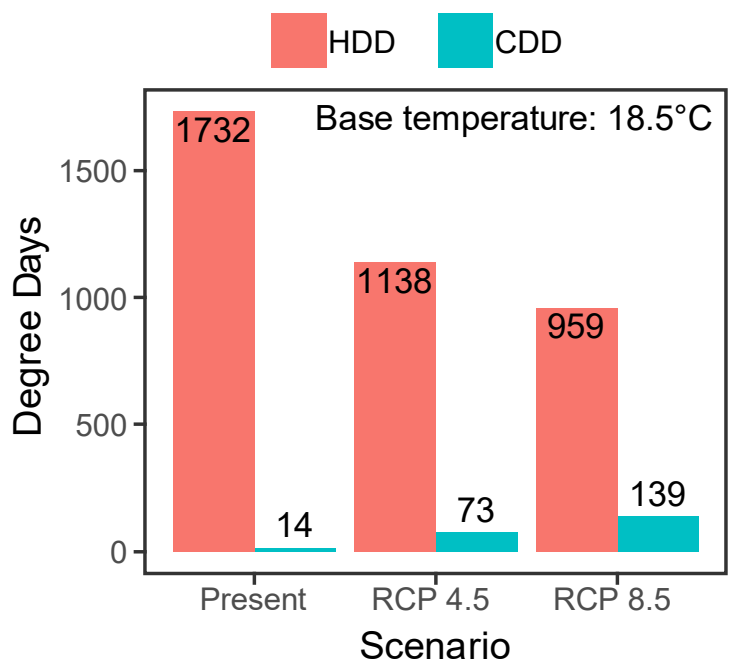

Figure 2: Heating and cooling degree days in present and future weather scenarios for San Francisco

\section{Base Building Energy Consumption}

To understand the optimization results, we also reviewed the base building energy consumption and how it changes due to the weather changes illustrated in Figures 1 and 2. Figure 3 breaks down the energy consumption by end use for the existing Whole Foods Store.

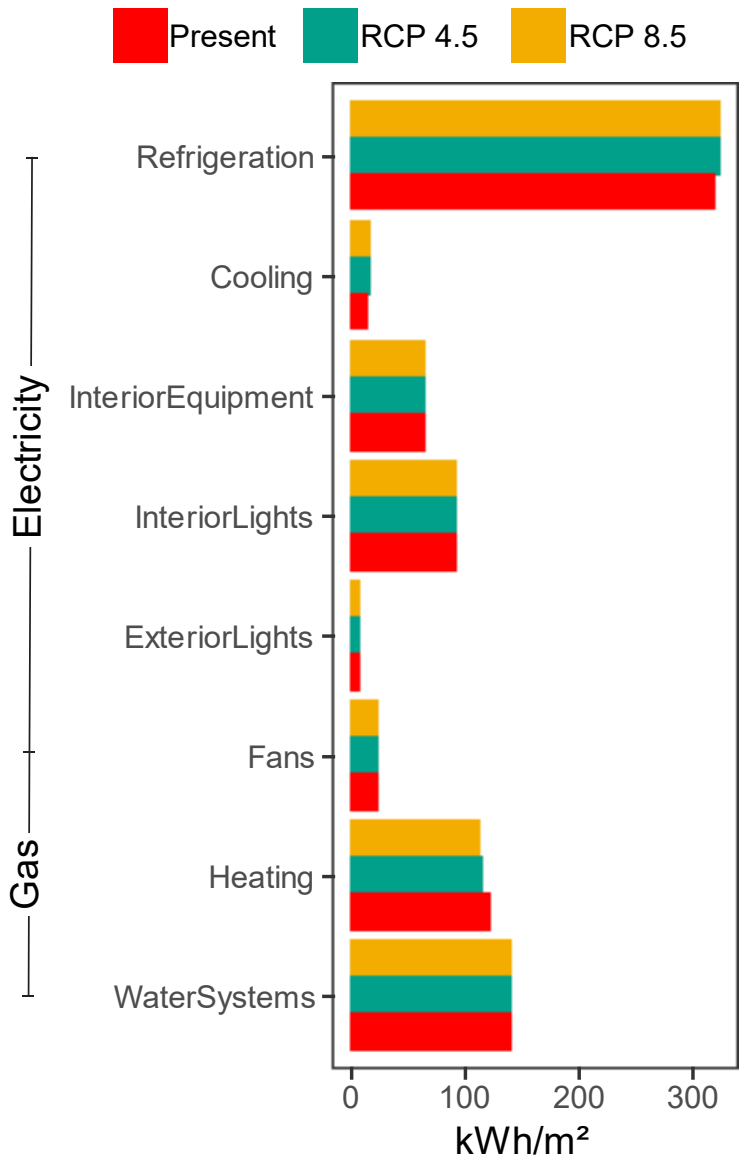

Figure 3: Energy consumption by end use for the existing Whole Foods grocery store in the present and two future weather scenarios

From Figure 3, we see that the store's energy consumption is dominated by refrigeration. It is also interesting to note that while most commercial buildings are cooling dominated due to high internal heat gains, grocery stores are generally heating dominated due to the cooling lost from refrigeration cases, as is also the case here. Due to the warming climate, we see an increase in energy consumption by refrigeration and cooling and a decrease in energy consumption by heating. Other end uses like interior equipment and interior lights are not affected by future weather. Nevertheless, reducing end uses seemingly independent of future weather indirectly impact space heating and cooling loads, and thus could affect the cost effectiveness of individual ECMs during computational optimization.

\section{Computational Optimization}

The GA is a stochastic process, so rather than review the results of a single optimal design, we reviewed the distribution of ECMs in the 250 best performing designs, which represents approximately $10 \%$ of simulated ECM 
combinations. We can then look for patterns in how frequently ECMs are selected or not selected to form an overall design recommendation.

Figure 4, at the end of this paper, compares the probability of the optimization selecting an individual ECM in the present and both future weather scenarios. While ECMs differ on the number of parametric variants, we considered an ECM as "selected" if the optimization included any variant. As a result, our results do not explore potential changes to the optimal ECM variant across weather scenarios. For visualization, we ordered ECMs by the probability of selection in the present.

From Figure 4, we can identify that there are many ECMs that the GA selected in the present and the future such as upgrade computers, replace rotisserie, heat pump water heater, and ensure plug loads off at night. These strategies often relate to directly reducing plug loads and are largely independent of weather, so it makes sense that they should be cost effective in both the present and future.

There are also many ECMs that the GA did not select in the present nor in either future weather scenario, such as installing windows on the $2^{\text {nd }}$ floor, insulate medium temperature piping, insulate roof, and solar air preheating. These strategies are associated with high capital costs relative to the savings in operational energy cost. In particular, installing new windows, insulation, and solar air preheating all benefit store heating, which is a decreasing portion of energy use in the future.

From Figure 4, we can also start to see that the likelihood of selecting some ECMs may also change between the present and either future scenarios, such as refrigeration heat recovery, which is nearly always selected in the present, but almost never selected by either future scenario.

Figure 5, at the end of this paper, highlights changes in the likelihood of selecting an individual ECM by subtracting the probability of selecting an ECM in the present from the probability of selecting that ECM in either future scenario. A positive value means that the probability of selecting that ECM increases in the future, while a negative value means the probability decreases.

Where some ECMs were almost never selected in the present, like upgrade RTUs, natural ventilation, and improving loading doors, these strategies are now almost always selected by both future weather scenarios. The performance of these strategies is directly tied to the exterior climate and their cost effectiveness improves as the weather warms.

It is particularly interesting that the energy model found natural ventilation to be cost effective. For cooling dominated building types, the efficiency of natural ventilation as a passive cooling strategy in many climates will likely decrease as the climate warms. However, as observed in Figure 3, grocery stores are generally heating dominated due to the large number of refrigeration cases. additionally, the location of this store in San Francisco provides a relatively cool starting point from which warming may increase the number of comfortable hours in parts of the city.
Another interesting result was a decrease in the frequency of solutions with refrigeration heat recovery via a desuperheater under both future climate scenarios. Reduced dependence on a desuperheater may indicate in a warmer climate there would be more extra heat than the one that is needed for heating or hot water. The finding highlights the need to evaluate interacting systems to determine the impact of future climate rather than isolating subsystems or individual building technology or ECM decisions.

\section{Procurement Approach}

An additional observation from the finding is that while there is overlap in many of the selected ECMs, designing optimally for the future requires either planning for the future today or planning to retrofit systems over time. Which approach is taken will vary by system depending on the expected life of each piece of equipment. Though not the focus of this study, each ECM can be ranked by expected life to prioritize when investments should be made.

For instance, rooftop HVAC unit replacement should occur every 15 years according to expected ASHRAE replacement schedules. This is also a case where choice of RTU upgrade varied between the present and future. Given the short life span of units, RTUs could be replaced today and optimally selected for near-term climate with the intent of re-evaluating the selection when the next purchase is required. By contrast, changes to the refrigeration system architecture or envelope improvements to facilitate natural ventilation are significant investments which are unlikely to be replicated until the next major renovation. Given that the choice of including these ECMs varied between present and future, a more detailed risk study may be required to ensure the correct investment is made initially to prepare the building changes in climate.

\section{Conclusion}

Our study finds that changes in future weather can tip the scales of decision making during the design process. Overall, we found that of the 57 ECMS we evaluated for energy and cost effectiveness, roughly 24 were effective at the present and 14 were ineffective in the present. Of those that were effective, 15 remained effective in the future, such as LED lights, while 9 became less effective in the future, such as refrigeration heat recovery. Of those that were ineffective, 4 remain ineffective, such as insulating walls, while 10 became more effective, such as upgrading rooftop units.

This shows that we should be careful with recommending ECMs that represent significant capital investments and whose payoff may decrease due to future weather changes, such refrigeration system architecture or envelope improvements. For ECMs with shorter service lives, likes mechanical equipment, it may be suitable to optimize for the present and reevaluate system performance at the time of future upgrades. Our study demonstrates the importance of evaluating performance with future weather projections. This will help ensure that buildings designed to be $\mathrm{ZNE}$ in the present can continue 
to achieve their performance goals throughout their service life, even with uncertainty in future weather

\section{Acknowledgements}

This work was supported in part through California Energy Commission grant GFO-15-308. The authors would also like to acknowledge the support of Tristam Coffin and Aaron Daly at Whole Foods Market, and Ben Brannon, Erica Levine, Russell Carr, and Ingrid Chaires at Arup.

\section{References}

Acha, S., Mavromatidis, G., Caritte, V., \& Shah, N.. (2013). Techno-Economical Technology Assessment for Operational Zero Carbon Supermarkets. Proceedings from ECOS 2013. Guilin (China), 16-19 July 2013.

Arup. (2012). The Technical Feasibility of Zero Net Energy Buildings in California, CALMAC Study IDPGE0326.01. kmg San Francisco (USA).

Best, R., \& Levine, E.. (2018). An Innovative Approach to Evaluating Energy Efficiency Measures for Zero Net Energy Supermarkets. Proceedings from 2018 ACEEE Summer Study on Energy Efficient Buildings. Pacific Grove (USA), 12-17 August 2018.

CARB. (2017). California's 2017 Climate Change Scoping Plan, The Strategy for Acheiving California's 2030 Greenhouse Gas Target. CARB. Sacramento (USA).

CEC. (2007). 2007 Integrated Energy Policy Report, CEC-100-2007-008-CMF. CEC. Sacramento (USA).

de Coninck, H., Revi, A., \& et al. (2018). Strengthening and Implementing the Global Response. In MassonDelmotte, V, Zhai, P., \& et al. Global Warming of $1.5^{\circ} \mathrm{C}$. World Meteorological Organization. Geneva (Switzerland).

Dickinson, R., \& Brannon, B. (2016). Generating Future Weather Files for Resilience. Proceedings from PLEA 2016 Los Angeles - $32^{\text {nd }}$ International Conference on Passive and Low Energy Architecture, Los Angeles (USA), 11-13 July 2016.

Evins, R. (2013). A Review of Computational Optimisation Methods Applied to Sustainable Building Design. Renewable and Sustainable Energy Reviews 22 (June), 230-45.

Hachem-Vermette, C., Cubi, E., \& Bergerson, J. (2016). Energy Performance of a Solar Mixed-Use Community. Sustainable Cities and Society 27 (November), 145-51.

O’Brien, W., Athienitis, A., \& Kesik, T. (2011). Parametric Analysis to Support the Integrated Design and Performance Modeling of Net Zero Energy Houses. ASHRAE Transactions 17, 945-960.

Pless, S., \& Torcellini, P. (2010). Net-Zero Energy Buildings: A Classification System Based on
Renewable Energy Supply Options, NREL/TP-55044586. NREL. Golden (USA).

Tuhus-Dubrow, D., \& Krarti, M. (2010). GeneticAlgorithm Based Approach to Optimize Building Envelope Design for Residential Buildings. Building and Environment 45 (7), 1574-81.

Wang, W., Zmeureanu, R., \& Rivard, H. (2005). Applying Multi-Objective Genetic Algorithms in Green Building Design Optimization. Building and Environment 40 (11), 1512-25. 


\begin{tabular}{|c|c|c|c|}
\hline Frequency & ECM & Present $\quad$ RCP 4.5 & RCP 8.5 \\
\hline \multirow{24}{*}{$\begin{array}{l}\text { Nearly always } \\
\text { selected in Present }\end{array}$} & Upgrade Computers & & \\
\hline & Insulate Glass & & \\
\hline & Replace Rotisserie & & \\
\hline & Heat Pump Water Heater & & \\
\hline & LED Store Lights & & \\
\hline & Case Occupancy Controls & & \\
\hline & Sales Occupancy Controls & & \\
\hline & Upgrade Walk-Ins & & \\
\hline & Enclose Refrigeration Cases & & \\
\hline & Automatic Door Closers on Walk-Ins & & \\
\hline & Upgrade Compressor Racks & & \\
\hline & Secondary Refrigeration Loop & & \\
\hline & Refrigeration Heat Recovery & & \\
\hline & Efficient Condenser & & \\
\hline & Add Doors to Walk-Ins with Curtains Only & & \\
\hline & Mechanical Subcooler & & \\
\hline & Improve Doors on Walk-Ins with Doors & & \\
\hline & Replace Back of House AC & & \\
\hline & Replace Ice Machines & & \\
\hline & Ensure Plug Loads Off at Night & & \\
\hline & $\mathrm{BOH}$ Occupancy Controls & & \\
\hline & Anti-Sweat Heater Controls & & \\
\hline & LED Exterior Lights & & \\
\hline & Enclose Warming Cases & & \\
\hline \multirow{19}{*}{$\begin{array}{l}\text { Sometimes } \\
\text { selected in the } \\
\text { Present }\end{array}$} & Widen Store Temperature Setpoints & & \\
\hline & Restroom Occupancy Controls & & \\
\hline & Enclose Refrigerated Bins & & \\
\hline & Consolidate Walk-Ins & & \\
\hline & Medium Temperature Floating Suction Head & & \\
\hline & Destratification Fans & & \\
\hline & Low Temperature Refrigerant Piping & & \\
\hline & HRV on Kitchen Exhaust & & \\
\hline & Upgrade Registers & & \\
\hline & Upgrade Refrigerated Table & & \\
\hline & Dishwasher Heat Recovery & & \\
\hline & Demand-Based Defrost on Walk-Ins & & \\
\hline & Low Flow Spray Valves & & \\
\hline & HRV on Restroom Exhaust & & \\
\hline & Natural Ventilation & & \\
\hline & Upgrade Conveyor Belt Motors & & \\
\hline & Mid-voltage DC Bus & & \\
\hline & Improve Loading Doors & & \\
\hline & Install Skylighting & & \\
\hline \multirow{14}{*}{$\begin{array}{c}\text { Nearly never } \\
\text { selected in Present }\end{array}$} & Upgraded Server Room AC & & \\
\hline & Variable Speed Kitchen Exhaust & & \\
\hline & Replace Once-Through Dipperwell & & \\
\hline & Widen Server Room Temperature Setpoints & & \\
\hline & Install Windows on 2nd Floor & & \\
\hline & Hot Water Recirculation & & \\
\hline & Demand Control Ventilation & & \\
\hline & Shade Front Façade & & \\
\hline & Insulate Medium Temp Refrigerant Piping & & \\
\hline & Upgrade RTUs & & \\
\hline & Insulate Walls & & \\
\hline & Insulate Roofs & & \\
\hline & Solar Air Preheating & & \\
\hline & Low Temperature Floating Suction Head & & \\
\hline
\end{tabular}

Figure 4: Comparison of probability of ECM selection between the present and future weather scenarios 


\begin{tabular}{|c|c|c|c|}
\hline Optimal Time & ECM & RCP 4.5 & RCP 8.5 \\
\hline \multirow{17}{*}{$\begin{array}{c}\text { Future but not } \\
\text { Present }\end{array}$} & Upgrade RTUs & & \\
\hline & Natural Ventilation & & \\
\hline & Improve Loading Doors & & \\
\hline & HRV on Restroom Exhaust & & \\
\hline & Mid-voltage DC Bus & & \\
\hline & Demand-Based Defrost on Walk-Ins & & \\
\hline & Install Skylighting & & \\
\hline & Hot Water Recirculation & & \\
\hline & Variable Speed Kitchen Exhaust & & \\
\hline & Shade Front Façade & & \\
\hline & Upgraded Server Room AC & & \\
\hline & Insulate Walls & & \\
\hline & Upgrade Refrigerated Table & & \\
\hline & Replace Once-Through Dipperwell & & \\
\hline & Consolidate Walk-Ins & & \\
\hline & Dishwasher Heat Recovery & & \\
\hline & Low Flow Spray Valves & & \\
\hline \multirow{21}{*}{$\begin{array}{c}\text { No change } \\
\text { between Present } \\
\text { and Future }\end{array}$} & HRV on Kitchen Exhaust & & \\
\hline & Demand Control Ventilation & & \\
\hline & Widen Server Room Temperature Setpoints & & \\
\hline & Insulate Medium Temp Refrigerant Piping & & \\
\hline & Upgrade Conveyor Belt Motors & & \\
\hline & Widen Store Temperature Setpoints & & \\
\hline & Low Temperature Floating Suction Head & & \\
\hline & BOH Occupancy Controls & & \\
\hline & Insulate Roofs & & \\
\hline & & & \\
\hline & Heat Pump Water Heater & & \\
\hline & LED Store Lights & & \\
\hline & Case Occupancy Controls & & \\
\hline & Sales Occupancy Controls & & \\
\hline & Upgrade Walk-Ins & & \\
\hline & Enclose Refrigeration Cases & & \\
\hline & Upgrade Compressor Racks & & \\
\hline & Secondary Refrigeration Loop & & \\
\hline & Efficient Condenser & & \\
\hline & Medium Temperature Floating Suction Head & & \\
\hline & Upgrade Computers & & \\
\hline \multirow{18}{*}{$\begin{array}{l}\text { Present but not } \\
\text { Future }\end{array}$} & Automatic Door Closers on Walk-Ins & & \\
\hline & Mechanical Subcooler & & \\
\hline & Install Windows on 2nd Floor & & \\
\hline & Ensure Plug Loads Off at Night & & \\
\hline & LED Exterior Lights & & \\
\hline & Enclose Warming Cases & & \\
\hline & Destratification Fans & & \\
\hline & Upgrade Registers & & \\
\hline & Enclose Refrigerated Bins & & \\
\hline & Replace Ice Machines & & \\
\hline & Replace Back of House AC & & \\
\hline & Low Temperature Refrigerant Piping & & \\
\hline & Add Doors to Walk-Ins with Curtains Only & & \\
\hline & Insulate Glass & & \\
\hline & Restroom Occupancy Controls & & \\
\hline & Anti-Sweat Heater Controls & & \\
\hline & Improve Doors on Walk-Ins with Doors & & \\
\hline & Refrigeration Heat Recovery & & \\
\hline
\end{tabular}

